\title{
A bizarre complication of shoulder arthroscopy
}

\section{Maurilio Bruno, Vito Lavanga, Emanuele Maiorano \& Valerio Sansone}

Knee Surgery, Sports Traumatology, Arthroscopy

\section{ISSN 0942-2056}

Knee Surg Sports Traumatol Arthrosc DOI 10.1007/s00167-013-2746-y

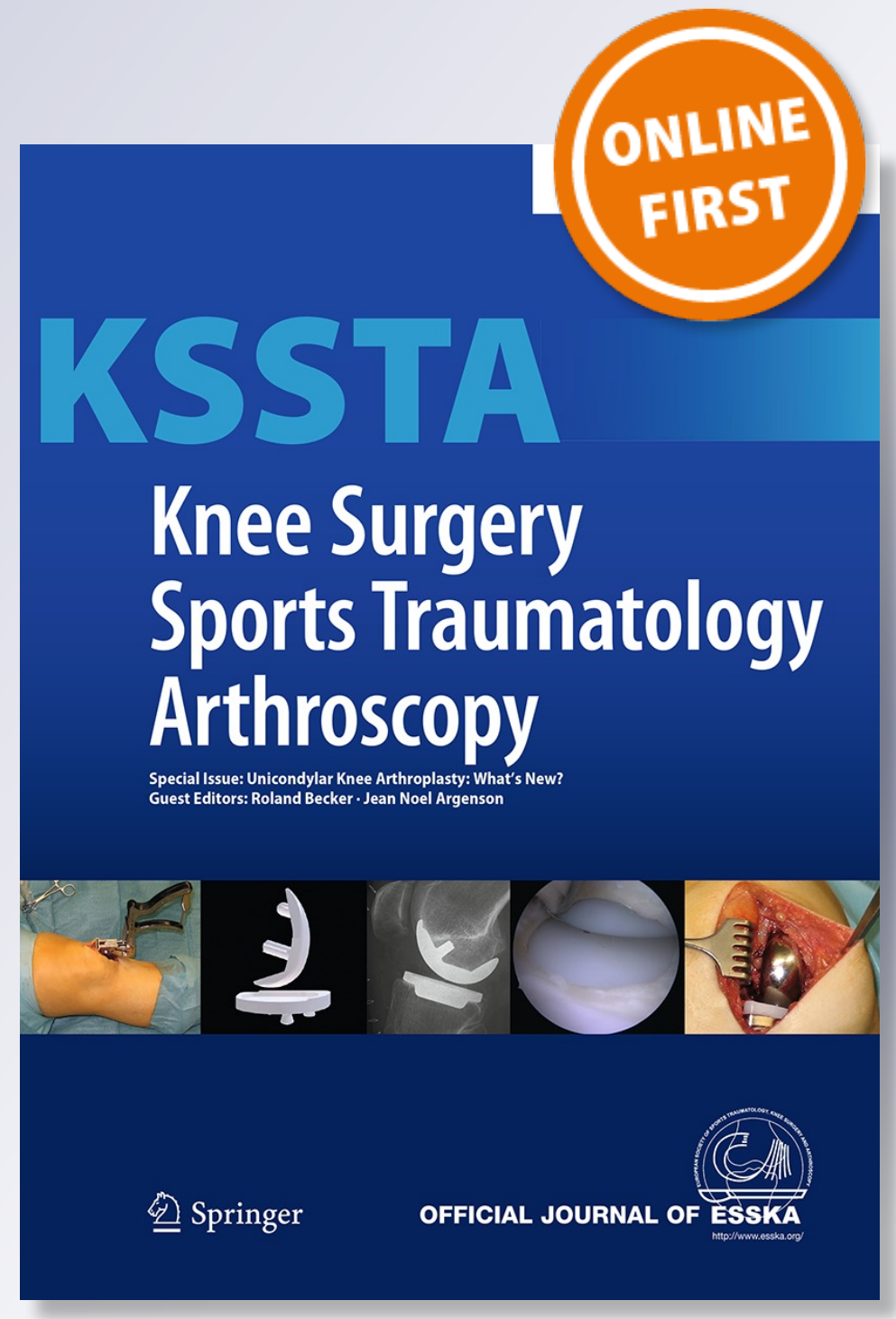

望 Springer 
Your article is protected by copyright and all rights are held exclusively by SpringerVerlag Berlin Heidelberg. This e-offprint is for personal use only and shall not be selfarchived in electronic repositories. If you wish to self-archive your article, please use the accepted manuscript version for posting on your own website. You may further deposit the accepted manuscript version in any repository, provided it is only made publicly available 12 months after official publication or later and provided acknowledgement is given to the original source of publication and a link is inserted to the published article on Springer's website. The link must be accompanied by the following text: "The final publication is available at link.springer.com". 


\title{
A bizarre complication of shoulder arthroscopy
}

\author{
Maurilio Bruno • Vito Lavanga • \\ Emanuele Maiorano $\cdot$ Valerio Sansone
}

Received: 21 May 2013/ Accepted: 23 October 2013

(C) Springer-Verlag Berlin Heidelberg 2013

\begin{abstract}
A case is presented of an unusual complication of shoulder arthroscopy, which was caused by incorrect location of the posterior portal, inducing neurapraxia of both the axillary and radial nerves, with significant clinical and functional sequelae. A subsequent open surgical neurolysis was required to restore normal nerve function. This type of lesion has not been reported previously.

Level of evidence $\mathrm{V}$.
\end{abstract}

Keywords Shoulder · Arthroscopy $\cdot$ Neurological complications · Neurolysis

\section{Introduction}

The complication rate following shoulder arthroscopy has been reported to be between 4.6 and $10.6 \%$ [2-5, 7, 9, 10]. Neurological complications are infrequent, with reported rates ranging from less than $3 \%[1,6]$ to less than $0.2 \%$ $[4,8,9]$. In most cases, these injuries are mild and result in transient paresthesia and hypoesthesia. However, more serious injuries with permanent damage have been described [6]. They usually are related to patient positioning and anaesthesia, although also portal placement is critical.

This case report describes a lesion of the posterior cord of the brachial plexus during the establishment of

M. Bruno · V. Lavanga · E. Maiorano · V. Sansone $(\bowtie)$ Istituto Ortopedico Galeazzi IRCCS, Via Galeazzi 4, 20161 Milan, Italy

e-mail: valerio.sansone@unimi.it

V. Lavanga · E. Maiorano - V. Sansone

Orthopaedic Department, Università degli Studi di Milano,

Via Festa del Perdono, 20122 Milan, Italy the posterior arthroscopic portal, which caused a functional impairment of both the axillary and the radial nerve. No other similar case has been reported in the Literature.

\section{Case report}

A 53-year-old man in general good health was admitted to our hospital complaining of intense left shoulder pain associated with a functional impairment of the left arm. The onset of symptoms occurred immediately after he underwent a shoulder arthroscopy of his left shoulder for a suspected subacromial impingement, 3 months earlier. The surgical report received from the operating hospital stated that the operation had been performed under general anaesthesia in lateral decubitus, with traction (amount unspecified). In addition to a persistent and intense pain, the patient complained of paresthesia along the posterior aspect of the arm and the dorsal aspect of the forearm and weakness of the whole upper limb. Clinical examination showed hypotrophy and weakness of the deltoid and trapezius muscles; elevation was possible to 90 degrees and abduction to 95 degrees. A partial left hand drop was also observed. It was also evident that the unique surgical scar of the posterior arthroscopic portal was more distal than usual (Fig. 1), although the anterior portal seemed positioned correctly. The disabilities of the arm, shoulder and hand score (DASH) was $36.4 \quad(0=$ no disability, $100=$ extreme disability).

Electroneuronography showed chronic neurological damage of the left axillary nerve with reduced potentials. The examination was incomplete as the radial nerve had not been tested. A colour Doppler ultrasound excluded any vascular disorder. 


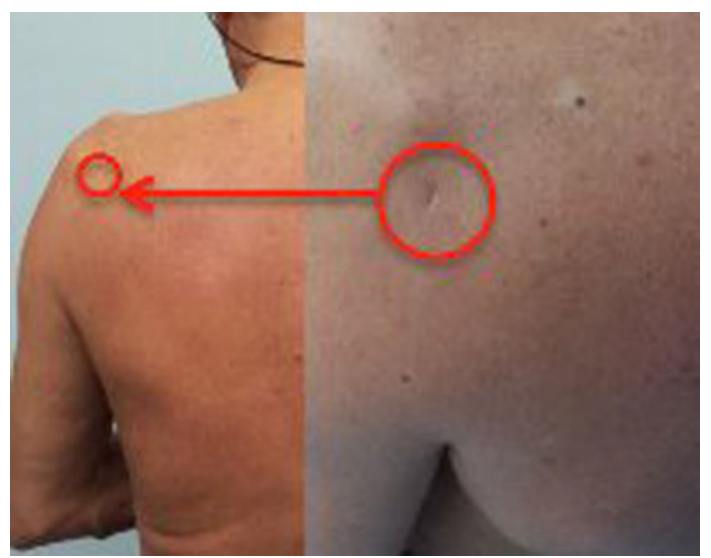

Fig. 1 Surgical scar of the posterior arthroscopic portal (red circle and arrow), located approximately $6 \mathrm{~cm}$ distal and $3 \mathrm{~cm}$ medial from the posterior-lateral corner of the acromion. The hypotrophy of trapezius and deltoid muscles is also evident

Then, a surgical neurolysis was planned. With the patient in the beach-chair position, a posterior open approach was conducted first. There was still an evident puncture hole in the fibres of the teres minor muscle which provided access to the quadrangular space (Fig. 2a), although the axillary nerve and the posterior circumflex artery resulted intact. An anterior deltopectoral access was then performed. On opening the clavipectoral fascia, there was an evident posterior-anterior tract that emerged near the brachial plexus. The posterior cord was surrounded by scar tissue that was carefully removed. After neurolysis, the nerve, although continuous, showed signs of a blunt contusion in its posterior aspect.

Post-operatively, a swift decrease in pain and a gradual recovery of muscle strength was observed. After 6 months, the patient has a nearly complete ROM of his left shoulder and is pain free. The post-operative DASH score is 2.3.

\section{Discussion}

The standard posterior arthroscopic portal of the shoulder is usually placed above the midline of the glenoid, approximately $1 \mathrm{~cm}$ medial and $1-2 \mathrm{~cm}$ inferior to the posterolateral corner of the acromion.

Wolf described a lower posterior portal, placed at the level of the equator of the posterior glenoid joint line [11]. The aim of this portal is to allow a better view of the anterior glenoid, useful during stabilization procedures. This portal, normally called the "central posterior", is created $1-2 \mathrm{~cm}$ medial and $3-4 \mathrm{~cm}$ distal to the posteriorlateral corner of the acromion.

In this case, the arthroscopicist probably aimed to establish a posterior central portal but he placed it too distally and failed to direct the arthroscopic trocar towards the coracoid process. After piercing the teres minor muscle fibres, the trocar probably missed the joint and entered the quadrangular space. This is an axillary space bounded by the teres minor muscle above, the teres major muscle below, the long head of the triceps brachial medially and the surgical neck of the humerus laterally. It transmits the axillary nerve and the posterior circumflex humeral artery. It is likely that the trocar continued unopposed along the scapular neck, finally hitting the posterior cord of the brachial plexus at the anterior aspect of the shoulder. During the revision surgery, when a pair of blunt scissors was cautiously introduced into the posterior opening and the tract was probed, the tip of the tool emerged anteriorly through the subscapularis muscle surprisingly easily, in close proximity to the infraclavicular portion of the brachial plexus (Fig. 2b). It seems reasonable to suppose that this had been the path of the arthroscopic trocar. This also accounts for the polyradicular nature of the neurological impairment observed; the damage was to the posterior cord
Fig. 2 a Open posterior approach showing the puncture hole in the teres minor muscle, presumably made by the arthroscopic trocar during the original surgery (scissors have been placed in the hole) $\mathbf{b}$ Open deltopectoral approach showing the puncture hole made by the arthroscopic trocar as it passed through the subscapularis muscle (scissors emerging from the hole)
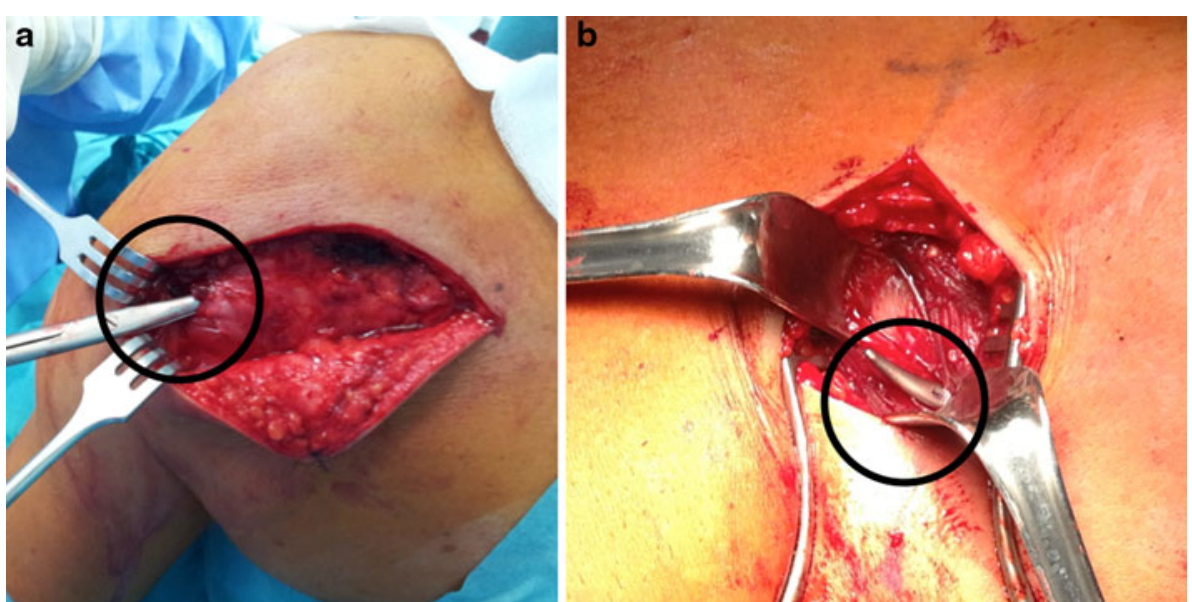
Fig. 3 Neurological structures of the brachial plexus, showing the site of the lesion

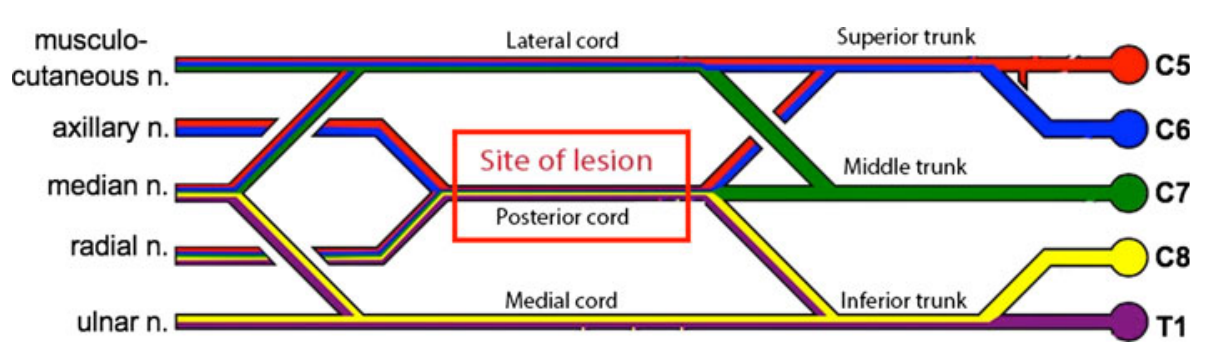

of the brachial plexus that terminates in the axillary and the radial nerves (Fig. 3).

The arthroscopy had been performed in lateral decubitus. It is difficult to say whether this was a contributing factor to the neurological damage that occurred. Very few studies have been conducted into how the position of the neurological structures may change in the lateral decubitus versus the beach-chair position, and they are mainly related to the axillary nerve [12]. However, it seems reasonable that arm abduction could play a role in pulling the infraclavicular portion of the brachial plexus closer to the glenohumeral joint.

\section{Conclusions}

From this case various conclusions can be drawn. When performing shoulder surgery, it is mandatory to have a sound knowledge of the nerve anatomy; this anatomy is not always constant and may change according to patient positioning. The standard posterior is a relatively secure portal for almost all arthroscopic procedures; a central posterior portal should only be used by experienced surgeons when a better view of the anterior glenoid is required. The central posterior approach should not be placed more than 3-4 cm distally from the posterolateral corner of the acromion and $2 \mathrm{~cm}$ medially, so as not to jeopardize the axillary nerve and the humeral posterior circumflex artery. A blunt trocar should be used and cautiously advanced towards the tip of the coracoid process. Finally, the neurovascular status of all patients should be checked before discharge.
Conflict of interest None.

\section{References}

1. Andrews JR, Carson WG (1983) Shoulder joint arthroscopy. Orthopedics 6:157-1162

2. Berjano P, González BG, Olmedo JF, Perez-España LA, Munilla MG (1998) Complications in arthroscopic shoulder surgery. Arthroscopy 14(8):785-788

3. Brislin KJ, Field LD, Savoie FH III (2007) Complications after arthroscopic rotator cuff repair. Arthroscopy 23(2):124-128

4. Committee on Complications of the Arthroscopy Association of North America (1986) Complications in arthroscopy: the knee and other joints. Arthroscopy 2(4):253-258

5. Curtis AS, Snyder SJ, Del Pizzo W, Friedman MJ, Ferkel RD, Karzel RP (1992) Complications of shoulder arthroscopy. Arthroscopy 8(3):395

6. Matthews LF, Zarins B, Michael RH et al (1985) Anterior portal selection for shoulder arthroscopy. Arthroscopy 1:33-39

7. Müller D, Landsiedl F (2000) Arthroscopy of the shoulder joint: a minimal invasive and harmless procedure? Arthroscopy 16(4):425

8. Ogilvie-Harris DJ, Wiley AM (1986) Arthroscopic surgery of the shoulder. a general appraisal. J Bone Joint Surg Br 68(2):201-207

9. Small NC (1988) Complications in arthroscopic surgery performed by experienced arthroscopists. Arthroscopy 4(3):215-221

10. Weber SC, Jain R (2002) Scalene regional anesthesia for shoulder surgery in a community setting: an assessment of risk. J Bone Joint Surg Am 84(5):775-779

11. Wolf EM (1989) Anterior portals in shoulder arthroscopy. Arthroscopy 5:201-208

12. Yoo JC, Kim JH, Ahn JH, Lee SH (2007) Arthroscopic perspective of the axillary nerve in relation to the glenoid and arm position: a cadaveric study. Arthroscopy 23(12):1271-1277 